\title{
Calculation of the Effective Permeability Tensor Used in the Naturally Fractured Reservoirs Simulation
}

\author{
Y.F. Liu \\ Xi'an shiyou university \\ Xi'an, P.R. China \\ L. Cao \\ Xi'an shiyou university \\ Xi'an, P.R. China
}

\author{
X. Wang \\ Petro China coal-bed methane company limited \\ XinZhou, P.R. China \\ Y.Z. Wang \\ Sinopec Southwest Oil \& Gas Company \\ P.R. China
}

\begin{abstract}
This paper presents a numerical model to calculate effective permeability tensor and to simulate the fluid flow in Naturally Fractured Reservoirs. This simulation includes three parts. A discrete fracture network is realized to capture the features of the target Naturally Fractured Reservoirs. The discrete fracture network is discretized into a limited number of grids whose effective permeabilities are calculated. Matching the production rate histories confirmed the correctness of the effective permeability tensor calculated in this way.
\end{abstract}

Keywords- natural fractured reservoir; effective permeability tensor; numerical simulation

\section{INTRODUCTION}

The development of Naturally Fractured Reservoirs provides opportunities for progress in reservoir numerical simulation techniques. Due to the complexity of reservoirs, Modeling of fluid flow in naturally fractured reservoirs is an important issue in the areas of petroleum engineering and hydrology.

There is still no determined method for Naturally Fractured Reservoirs simulation. Dual porosity and discrete fracture models are the main approaches to simulate fluid flow in naturally fractured reservoirs. $[1,2]$.

Currently, this thesis is aimed to calculate the effective permeability tensor and to simulate the fluid flow in Naturally Fractured Reservoirs. There are for Naturally Fractured Reservoirs simulation: the Equivalent Continuum Model (ECM), the Discrete Fracture Network Model (DFNM) and the Hybrid Model, all of which can be divided into deterministic model and stochastic model according to the inputs. The ECM is appropriate to filed-scale reservoir simulation while the DFNM is well known for its ability to describe heterogeneity of local-scale regions. The equivalent medium model presented in this work is a hybrid model who takes advantages of both the ECM and DFNM.

\section{MATHEMATICAL FORMULATION}

In this formulation, effective permeability is described as a full tensor that relates the average pressure gradient $\nabla \mathrm{P}$ to the average fluid velocity $\mathrm{V}$ as:

$$
\mathrm{V}=-\mathrm{K} \nabla \mathrm{P}
$$

Where $\mathrm{K}$ is the local permeability tensor describing the directional effect of fracture or set of fractures on fluid flow. The effective permeability tensor needs to be symmetric and positive definite in order to have a physical meaning, i.e. $\mathrm{k}_{\mathrm{xy}}=\mathrm{k}_{\mathrm{yx}}$ and $\left(\mathrm{k}_{\mathrm{xx}} \mathrm{k}_{\mathrm{yy}} \geq \mathrm{k}_{\mathrm{xy}}{ }^{2}, \mathrm{k}_{\mathrm{xx}}>0, \mathrm{k}_{\mathrm{yy}}>0\right)$.[3].

In the above definitions, $\mathrm{k}_{\mathrm{xx}}$ and $\mathrm{k}_{\mathrm{yy}}$ are diagonal terms and $\mathrm{k}_{\mathrm{xy}}$ and $\mathrm{k}_{\mathrm{yx}}$ are off-diagonal terms of permeability tensor $\mathrm{K}$. Assuming that fluid flow in matrix and fracture obeys Darcy's law and cubic law,[4], respectively, the governing equations of fluid flow in a two-dimensional reservoir are the following:[5].

$$
\begin{aligned}
& \text { Matrix: } \\
& \frac{\partial}{\partial \mathrm{x}}\left(\mathrm{k}_{\mathrm{xx}} \frac{\partial \mathrm{p}}{\partial \mathrm{x}}+\mathrm{k}_{\mathrm{xy}} \frac{\partial \mathrm{p}}{\partial \mathrm{y}}\right)+\frac{\partial}{\partial \mathrm{y}}\left(\mathrm{k}_{\mathrm{yx}} \frac{\partial \mathrm{p}}{\partial \mathrm{x}}+\mathrm{k}_{\mathrm{yy}} \frac{\partial \mathrm{p}}{\partial \mathrm{y}}\right)+\mathrm{Q}_{\mathrm{m}}=0 \\
& \text { Fracture: } \\
& \qquad \frac{\partial}{\partial \mathrm{L}}\left(\mathrm{k}_{\mathrm{f}} \frac{\partial \mathrm{p}}{\partial \mathrm{L}}\right)+\mathrm{Q}_{\mathrm{f}}+\mathrm{q}_{\mathrm{ff}}=0
\end{aligned}
$$

Where $\mathrm{k}_{\mathrm{f}}=7.842 \times 10^{12} \cdot \mathrm{h}^{2}, \mathrm{~h}$ is fracture aperture, $\mathrm{L}$ is onedimensional coordinate, and subscripts $\mathrm{m}$ and $\mathrm{f}$ represent matrix and fracture, respectively. Terms $Q_{m}$ and $Q_{f}$ represent flow interaction between the fracture and matrix. $\mathrm{q}_{\mathrm{ff}}$ is flow at the point of intersection by intersecting fractures .Matrix permeability is assumed to be constant in horizontal and vertical directions as in a homogenious system.

For notation simplicity, we consider the above equations in general form of a linear Poisson's equation in domain A as follows:

$$
\nabla \cdot(\mathrm{k} \nabla \mathrm{p}(\mathrm{x}))+\mathrm{Q}(\mathrm{x})=0 \text { in } \mathrm{A},
$$

Where $\mathrm{k}$ denotes the permeability, $\mathrm{x}=(\mathrm{x} 1, \mathrm{x} 2)$ is an arbitrary point in interior of $\mathrm{A}$ and $\mathrm{Q}(\mathrm{x})$ characterizes the source term associated with interior domain $\mathrm{A}$. In case, $\mathrm{A}$ is a medium or long fracture, the term $\mathrm{Q}(\mathrm{x})$ is defined as $\left(\mathrm{Q}_{\mathrm{f}}+\mathrm{q}_{\mathrm{ff}}\right)$. The boundary integral equation for the above equation pertaining to an arbitrary point $\xi=\left(\xi_{1}, \xi_{2}\right)$ is written as:

$$
\begin{array}{r}
\mathrm{c}(\xi) \mathrm{p}(\xi)=\int_{\mathrm{S}} \mathrm{G}(\mathrm{x}, \xi) \mathrm{v}(\mathrm{x}) \mathrm{ds}(\mathrm{x})-\int_{\mathrm{S}} \mathrm{F}(\mathrm{x}, \xi) \mathrm{p}(\mathrm{x}) \mathrm{ds}(\mathrm{x})+ \\
\int_{\mathrm{A}} \mathrm{Q}(\mathrm{x}) \mathrm{G}(\mathrm{x}, \xi) \mathrm{dA}(\mathrm{x})
\end{array}
$$

Where $v=k \nabla p \bar{n} . \bar{n}$ is the exterior normal vector of $S$ at $\xi$, and $\mathrm{S}$ is the boundary of domain $\mathrm{A} . \mathrm{G}$ is the fundamental 
solution, $F=k \frac{\partial G}{\partial n}$, which is its corresponding flux, and $\mathrm{Q}(\mathrm{x})=\sigma(\mathrm{x}-\xi)$, which is the Dirac delta function. The coefficient $c(\xi)$, which is a function of the internal angle of the boundary $S$ at point $\xi$, equal to 1 if $\xi \in A, 0$ if $\xi \notin A \cup S$ and $\frac{\alpha}{2 \pi}$ if $\xi \in S$, where $\propto$ denotes the interior angle of $S$ at $\xi(\propto$ equals $\pi$ at a smooth point on $S)$.[6].

For short fractures and the part of matrix which is not affected by fluid flow in medium and long fractures, the source term $\int_{\mathrm{A}} \mathrm{QGdA}$ is neglected and $\mathrm{Eq}$.(5) is rewritten as :

$$
\mathrm{c}(\xi) P(\xi)=\int_{\mathrm{S}} \mathrm{G}(\mathrm{x}, \xi) \mathrm{v}(\mathrm{x}) \mathrm{ds}(\mathrm{x})-\int_{\mathrm{S}} \mathrm{F}(\mathrm{x}, \xi) \mathrm{pds}(\mathrm{x})
$$

The interface boundary conditions for short fractures are expressed as:

$$
\mathrm{P}_{\mathrm{mi}}=\mathrm{P}_{\mathrm{fi}} \text {, and } \mathrm{v}_{\mathrm{mi}}=-\mathrm{v}_{\mathrm{fi}}
$$

Boundary conditions along the medium and long fracture boundaries are written as:

$$
\begin{gathered}
\mathrm{P}_{\mathrm{fi}}=\mathrm{P}_{\mathrm{av}} \\
\mathrm{v}_{\mathrm{mi}}{ }^{+}-\mathrm{v}_{\mathrm{mi}}{ }^{-}=\mathrm{Q}_{\mathrm{i}}
\end{gathered}
$$

Where $\quad \mathrm{v}_{\mathrm{mi}}{ }^{+}=\overrightarrow{\mathrm{v}}_{\mathrm{m}}{ }^{+} \cdot \overrightarrow{\mathrm{n}}, \mathrm{v}_{\mathrm{mi}}{ }^{-}=\overrightarrow{\mathrm{v}}_{\mathrm{m}}{ }^{-} \cdot \overrightarrow{\mathrm{n}}, \mathrm{P}_{\mathrm{av}}$ is the average pressure inside the fracture and $\mathrm{p}_{\mathrm{fi}}$ represents the pressure along the fracture boundaries.$\vec{v}_{\mathrm{m}}{ }^{+}$and $\overrightarrow{\mathrm{v}}_{\mathrm{m}}{ }^{-}$are velocities on the opposite nodes on the fracture faces and $Q_{i}$ deponds on the source strength of the fracture faces and represents the flow interaction between the matrix $\mathrm{m}$ and fracture I . Matrix pressure on the common matrix-fracture interface and on the exterior boundaries of the area around the fracture are unknown and will be calculated by applying the periodic boundary condition during the solution process.

The periodic boundary condition is considered for nodes along the block boundaries, which requires to treat all fracture edges as being inside the grid block. [7]. Assume that $\Gamma_{1}$ and $\Gamma_{2}$ are two opposite faces of the grid-block in the $\mathrm{x}_{2}$ direction and $\Gamma_{3}$ and $\Gamma_{4}$ are two opposite faces of the gridblock in the $\mathrm{x}_{1}$ direction .Pressure at an arbitrary point $\mathrm{x}=\left(\mathrm{x}_{1}\right.$, $\mathrm{x}_{2}$ ) in the grid-block can be expressed as :

$$
\mathrm{p}(\mathrm{x})=\mathrm{p}_{0}+\mathrm{J}\left(\mathrm{x}-\mathrm{x}_{0}\right)
$$

Where $x_{0}$ is the center of the region under consideration, $\mathrm{p}_{0}$ is the pressure at $\mathrm{x}_{0}$, and $\mathrm{j}=\left(\mathrm{j}_{1}, \mathrm{j}_{2}\right)$ is the local pressure gradient . The periodic boundary conditions over the unit cell are written as:

$$
\begin{gathered}
\mathrm{p}\left(\mathrm{x}_{1}, 0\right)=\mathrm{p}\left(\mathrm{x}_{1}, 1\right)-\mathrm{j}_{2} \text { on } \Gamma_{1} \text { and } \Gamma_{3} \\
\mathrm{v}\left(\mathrm{x}_{1}, 0\right) \overrightarrow{\mathrm{n}_{1}}=-\mathrm{v}\left(\mathrm{x}_{1}, 1\right) \cdot \overrightarrow{\mathrm{n}_{2}} \text { on } \Gamma_{1} \text { and } \Gamma_{3} \\
\mathrm{p}\left(0, \mathrm{x}_{2}\right)=\mathrm{p}\left(1, \mathrm{x}_{2}\right)-\mathrm{j}_{1} \text { on } \Gamma_{2} \text { and } \Gamma_{4} \\
\mathrm{v}\left(0, \mathrm{x}_{2}\right) \cdot \overrightarrow{\mathrm{n}_{2}}=-\mathrm{v}\left(1, \mathrm{x}_{2}\right) \cdot \overrightarrow{\mathrm{n}_{4}} \text { on } \Gamma_{2} \text { and } \Gamma_{4}
\end{gathered}
$$

A constant pressure difference in the $\mathrm{x} 1$ direction and a zero pressure difference in the $\mathrm{x} 2$ direction $\left(\mathrm{j}_{1} \neq 0\right.$ and $\mathrm{j}_{2}=0$ )are applied to calculate the first two terms of permeability tensor $\mathrm{k}_{\mathrm{xx}}$ and $\mathrm{k}_{\mathrm{yx}}$. The remaining two components of permeability tensor, $\mathrm{k}_{\mathrm{xy}}$ and $\mathrm{k}_{\mathrm{yy}}$ can be calculated in the same way by carrying the direction of pressure gradient to $\left(\mathrm{j}_{1}=0\right.$ and $\left.\mathrm{j}_{2} \neq 0\right)$

\section{APPLICATION}

There is a Naturally Fractured Reservoir called JA field located in China. We have built the structural modeling after collation of the data. The Petrophysical modeling was built with the method of Sequential Gaussian simulation. In this method, we got the curve of the history matching in Fig. 1.

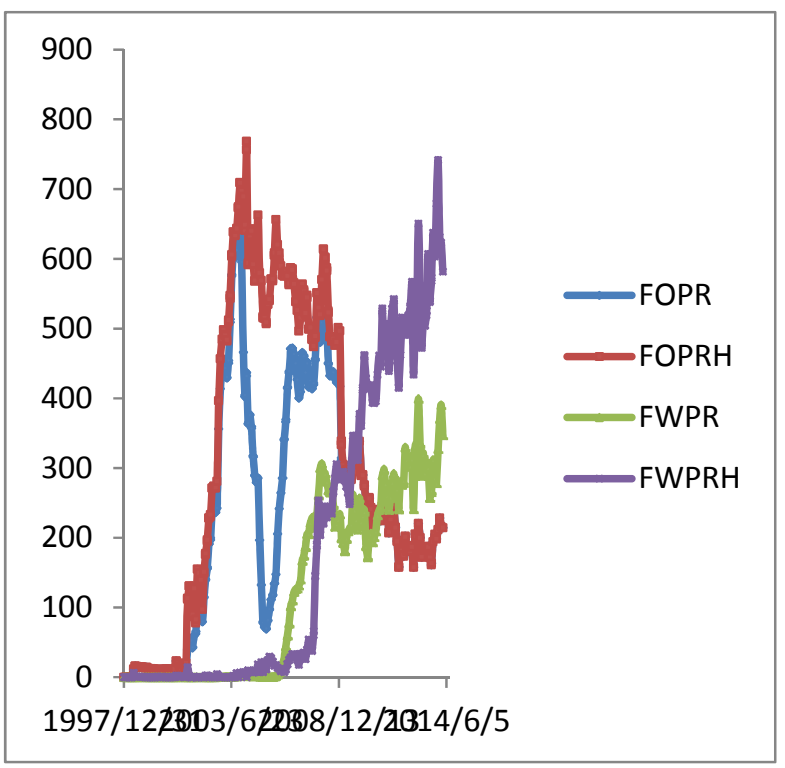

FIGURE I. HISTORY MATCHING CURVE WITH THE METHOD OF SEQUENTIAL GAUSSIAN SIMULATION.

In this curve, we can see the red line means the Field Oil Production Rate History, the blue one is the Field Oil Production Rate, the green one means the Field Water Production Rate, the purple line means the Field Water Production Rate History. We can get the information that the history matching effect is not ideal. The oil production at 2005 and the water production at the 2010 is lower than the date of history in the method of the Sequential Gaussian simulation.

Another property modeling was modified with the method of effective permeability tensor. We got the history matching curve in Fig. 2. 


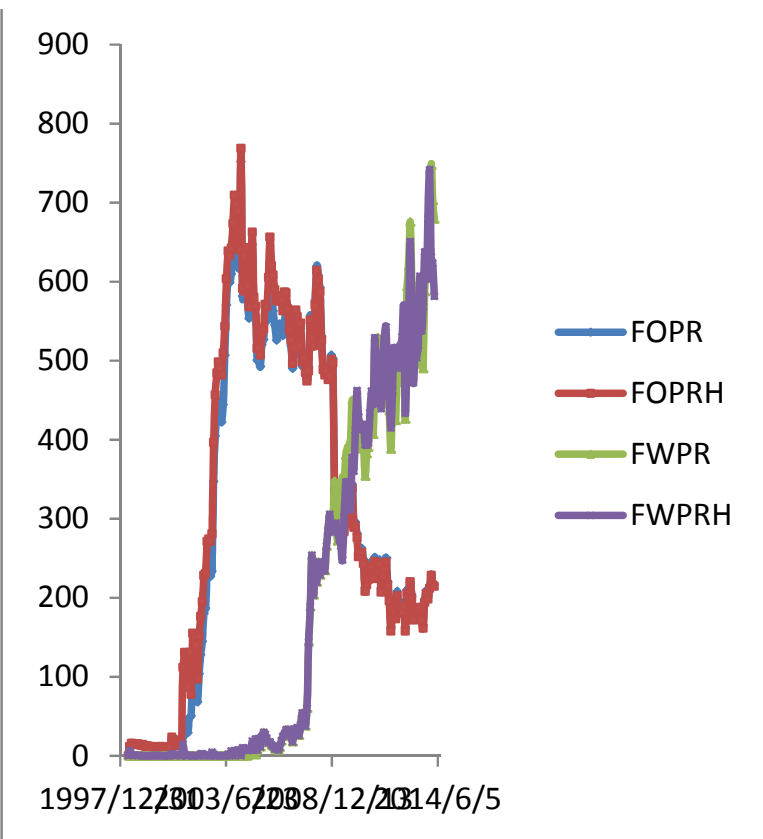

FIGURE II. HISTORY MATCHING CURVE WITH THE METHOD OF THE EFFECTIVE PERMEABILITY TENSOR.

Obviously, this history matching curve is better than the last one, It means the method of the effective permeability tensor is one of the best ways for the reservoirs simulation.

\section{CONCLUSIONS}

A model has been developed to calculate effective permeability tensor in naturally fractured reservoirs. Fluid flow through matrix, fracture and flow interaction between matrix and fracture are considered. From the results of this method following conclusions can be drawn:

1) The model presented in this study is able to calculate permeability tensor of naturally fractured reservoirs by considering the fact that the porous matrix, fractures of different sizes, (isolated), and interconnected fractures all contribute to the permeability of the reservoir.

2) Application of the effective permeability tensor method on the property modeling, the history matching curve has become better than the Sequential Gaussian Simulation. Its correctness had been confirmed.

\section{REFERENCE}

[1] Kazemi H.,Merril,L.S.,Porterfield ,K.L., and Zeman ,P.R.: "Numerical simulation of water-oil flow in naturally fractured reservoirs" Society of petroleum Engineering Journal , pp317-326, 1976.

[2] Van Golf-Racht,T.D.."Fundamental of fractured reservoir engineering", Elsevier ,1982.

[3] Durlofsky,L.J.:"Numerical calculation of equivalent grid block permeability tensors for heterogeneous porous media," Water Resources Research,1991.27(5):pp 699-708

[4] Binder,R.C. : "Advanced fluid mechanics", Englewood Cliffs, NJ: Prentice-Hall,1958

[5] Park,Y.C.,Sung,W.M.,Kim,S.J.:"Development of a FEM reservoir model equipped woth an effective permeability tensor and its application to naturally fractured reservoirs," Energy Sources,24,pp
531-542,2002 Rudolphi,T.,Petersen,V.: Advances in Engineering Software, 9(1),pp 7-28,1987.

[6] Rudolphi,T.,Petersen,V.:"Advances in Engineering Software", 9(1), pp 7-28, 1987

[7] Lough,M.F.,S.H. Lee , and J.Kamath :"An efficient boundary integral formulation for flow through fractured porous media , Journal of Computational Physics ,1998.143:p.462-483 